\title{
Development of the Korea-Polyenvironmental Risk Score for Psychosis
}

\author{
Eun-Jin Jeon', Shi-Hyun Kang ${ }^{2}$, Yan-Hong Piao ${ }^{3}$, Sung-Wan Kim², Jung-Jin Kim5, Bong-Ju Lee ${ }^{6}$, \\ Je-Chun $\mathrm{Yu}^{7}$, Kyu-Young Lee ${ }^{8}$, Seung-Hee Won ${ }^{9}$, Seung-Hwan Lee ${ }^{10}$, Seung-Hyun Kim ${ }^{11}$, Eui-Tae Kim ${ }^{12}$, \\ Clara Tammy Kim ${ }^{13}$, Dominic Oliver ${ }^{14,15}$, Paolo Fusar-Poli ${ }^{14,15,16}$, Fatima Zahra Rami ${ }^{3}$, and Young-Chul Chung ${ }^{1,3,17} \bowtie$ \\ 1'Department of Psychiatry, Jeonbuk National University Hospital, Jeonju, Republic of Korea \\ ${ }^{2}$ Department of Social Psychiatry and Rehabilitation, National Center for Mental Health, Seoul, Republic of Korea \\ ${ }^{3}$ Department of Psychiatry, Jeonbuk National University Medical School, Jeonju, Republic of Korea \\ ${ }^{4}$ Department of Psychiatry, Chonnam National University Medical School, Gwangju, Republic of Korea \\ ${ }^{5}$ Department of Psychiatry, The Catholic University of Korea, Seoul St. Mary's Hospital, Seoul, Republic of Korea \\ ${ }^{6}$ Department of Psychiatry, Inje University Haeundae Paik Hospital, Inje University College of Medicine, Busan, Republic of Korea \\ ${ }^{7}$ Department of Psychiatry, Eulji University School of Medicine, Eulji University Hospital, Daejeon, Republic of Korea \\ ${ }^{8}$ Department of Psychiatry, Nowon Eulji Medical Center, Eulji University, Seoul, Republic of Korea \\ ${ }^{9}$ Department of Psychiatry, School of Medicine, Kyungpook National University, Daegu, Republic of Korea \\ ${ }^{10}$ Department of Psychiatry, Inje University Ilsan Paik Hospital, Goyang, Republic of Korea \\ ${ }^{11}$ Department of Psychiatry, Korea University College of Medicine, Guro Hospital, Seoul, Republic of Korea \\ ${ }^{12}$ Department of Psychiatry, Seoul National University Bundang Hospital, Seongnam, Republic of Korea \\ ${ }^{13}$ Institute of Life and Death Studies, Hallym University, Chuncheon, Republic of Korea \\ ${ }^{14}$ Early Psychosis: Interventions and Clinical Detection (EPIC) Lab, Department of Psychosis Studies, Institute of Psychiatry, \\ Psychology and Neuroscience, King's College London, London, United Kingdom \\ ${ }^{15} \mathrm{OASIS}$ Service, South London and the Maudsley NHS Foundation Trust, London, United Kingdom \\ ${ }^{16}$ Department of Brain and Behavioural Sciences, University of Pavia, Pavia, Italy \\ ${ }^{17}$ Research Institute of Clinical Medicine of Jeonbuk National University-Biomedical Research Institute of Jeonbuk National University Hospital, \\ Jeonju, Republic of Korea
}

Objective Comprehensive understanding of polyenvironmental risk factors for the development of psychosis is important. Based on a review of related evidence, we developed the Korea Polyenvironmental Risk Score (K-PERS) for psychosis. We investigated whether the K-PERS can differentiate patients with schizophrenia spectrum disorders (SSDs) from healthy controls (HCs).

Methods We reviewed existing tools for measuring polyenvironmental risk factors for psychosis, including the Maudsley Environmental Risk Score (ERS), polyenviromic risk score (PERS), and Psychosis Polyrisk Score (PPS). Using odds ratios and relative risks for Western studies and the "population proportion" (PP) of risk factors for Korean data, we developed the K-PERS, and compared the scores thereon between patients with SSDs and HCs. In addition, correlation was performed between the K-PERS and Positive and Negative Syndrome Scale (PANSS).

Results We first constructed the "K-PERS-I," comprising five factors based on the PPS, and then the "K-PERS-II" comprising six factors based on the ERS. The instruments accurately predicted participants' status (case vs. control). In addition, the K-PERS-I and -II scores exhibited significant negative correlations with the negative symptom factor score of the PANSS.

Conclusion The K-PERS is the first comprehensive tool developed based on PP data obtained from Korean studies that measures polyenvironmental risk factors for psychosis. Using pilot data, the K-PERS predicted patient status (SSD vs. HC). Further research is warranted to examine the relationship of K-PERS scores with clinical outcomes of psychosis and schizophrenia.

Psychiatry Investig 2022;19(3):197-206

Keywords Polyenvironmental risk factor; Psychosis; Prediction.

Received: October 14, 2021 Revised: December 18, 2021 Accepted: December 26, 2021

$\triangle$ Correspondence: Young-Chul Chung, MD, PhD

Department of Psychiatry, Jeonbuk National University Medical School, Baekje-daero, Deokjin-gu, Jeonju 54896, Republic of Korea

Tel: +82-63-250-2185, Fax: +82-63-275-3157, E-mail: chungyc@jbnu.ac.kr

(c) This is an Open Access article distributed under the terms of the Creative Commons Attribution Non-Commercial License (https://creativecommons.org/licenses/by-nc/4.0) which permits unrestricted non-commercial use, distribution, and reproduction in any medium, provided the original work is properly cited. 


\section{INTRODUCTION}

Schizophrenia (SZ), one of the most detrimental and common psychiatric disorders, has an annual incidence of approximately $0.015 \%{ }^{1}$ It often has a devastating impact on patients' quality of life; about two-thirds of patients with SZ exhibit a chronic course characterized by relapse, ${ }^{2,3}$ and SZ patients have two- to threefold higher mortality compared to the general population, corresponding to a 10- to 25-year reduction in life expectancy. The heritability of SZ, i.e., the genetic contribution to phenotypic variance, is high, ranging between $41 \%$ and $87 \%{ }^{5-7}$ However, attempts to discover genes directly associated with SZ have been thwarted by a lack of replication of study findings. A recent genome-wide association study (GWAS) identified 108 loci associated with SZ. ${ }^{8}$ These variants together explained only a small proportion $(7 \%-36 \%)$ of the variance in liability, suggesting "missing heritability." ${ }^{90}$ Possible causes for the missing heritability of SZ include rare copy number variants, non-additive genetic effects (dominance and epistasis), gene-environment interactions, and environmental factors. Epidemiological studies have suggested that a variety of environmental factors, including prenatal infection/immune activation, paternal age, malnutrition, hypoxia-related obstetric complications, childhood/adolescence trauma and cannabis use are associated with an increased risk of developing SZ. ${ }^{11,12}$

Given the small proportion of the variance explained by individual single nucleotide polymorphisms (SNPs), the polygenic risk score (PRS), a weighted sum of the number of risk alleles in individuals, is now considered as a valid alternative approach and has been widely applied in research studies. A similar approach has been employed to predict conversion to psychosis by aggregating environmental risk factors. Intervention prior to the full manifestation of a disorder may delay or even prevent the onset of psychosis; early identification of those at high risk of psychosis is thus of great importance. Three tools using the aggregate score for multiple environmental risk factors have been developed: the polyenviromic risk score (PERS), ${ }^{13}$ Maudsley Environmental Risk Score (ERS) ${ }_{1}^{14}$ and Psychosis Polyrisk Score (PPS). ${ }^{15,16}$ The environmental risk factors included in these tools differ. The PERS has nine risk factors (winter or spring birth, urbanicity, cannabis use, advanced paternal age, obstetric and perinatal complications, history of physical abuse, sexual abuse, neglect, and loss of a parent/parental separation), the ERS has six (ethnic minority status, urbanicity, high paternal age, obstetric complications, cannabis use, and childhood adversity), and the PPS has sixteen (childhood trauma, ethnicity, immigration, premorbid intelligence quotient (IQ), nonright handedness, olfactory identification ability, clinical high risk for psychosis, urbanicity, etc). Only the PERS score was shown to be significantly correlated with conversion to psychosis in individuals with familial high risk of SZ. ${ }^{13}$ To apply these tools to populations in other countries with different cultures, the appropriateness of the included risk factors must be considered. The present study was undertaken to construct a Korean version of the PERS (K-PERS). After reviewing the evidence, we selected appropriate environmental risk factors for the K-PERS and calculated new risk scores based on the proportions of risk factors (exposure) in the Korea population. Pilot data from the K-PERS were compared between patients with schizophrenia spectrum disorders (SSDs) and healthy controls (HCs).

\section{METHODS}

\section{Search strategy for relevant literature}

Potentially relevant studies were identified by a comprehensive search of the PubMed, Embase, and PsychINFO electronic databases. Terms related to environmental risk in general, or to each putative risk factor (i.e., paternal age OR parental socioeconomic status [SES] OR pregnancy complication OR obstetric complications OR urbanicity OR child adversity/trauma/abuse OR cannabis/substance use OR recent life events) were combined with psychosis OR psychotic disorders OR SZ. The searches were limited to studies related to the ERS, PERS, and PPS, which measure multiple environmental risk factors, and to systematic reviews or meta-analyses of studies of putative risk factors. To determine the "population proportion" (PP) of risk factors in Korea, annual or survey reports issued by government-affiliated agencies (Ministry of Health and Welfare, Statistics Korea, Korea Land and Housing Corporation) were searched.

\section{Development of the K-PERS}

The ERS and PPS scores were estimated by scaling the odds ratios (ORs) or relative risks (RRs) with PP for each risk factor, whereas the PERS score was obtained by simply summing the ORs of the risk factors. We assumed that the former two tools may provide more valid estimates of the risk factors. Thus, we decided to consider the appropriateness only of the risk factors included in those two tools. Members of the Korea Early Psychosis Study (KEPS) team reviewed the risk factors included in the two tools. We decided that it would not yet be appropriate to include two factors, cannabis use and immigrant/ethnic minority status, in a tool designed for Koreans, although both factors are becoming increasingly important social issues in Korea. Also, premorbid IQ, olfactory identification ability, and pollution were considered to be impractical for clinical use. Paternal age, parental SES, and adult life events were not included in the original PPS ${ }^{15}$ but were adopted for the extended 
version. ${ }^{16}$ In the ERS, parental SES and recent life events were not included. However, we decided to include them given the associations of psychosis with social class ${ }^{17}$ and life events, ${ }^{18}$ as well as their potential clinical utility. As we found no large-scale Korean epidemiologic studies investigating relationships between environmental risk factors and psychosis, we decided to use ORs and relative risks (RRs) from Western studies, and the PP of risk factors for Korean data, to devise the K-PERS. The formulas for calculating scores based on OR, RR and PP data are fully described in the original PPS ${ }^{15}$ and ERS ${ }^{14}$ publications. The K-PERS questionnaire (Supplementary Material in the online-only Data Supplement) was developed to evaluate six risk factors: paternal age, parental SES, obstetric complications, urbanicity, childhood trauma, and adult life events. Cutoffs were applied to some risk factors, such as paternal age, parental SES, birth weight, and urbanicity, whereas childhood trauma and adult life events rated as moderate or higher were assessed in terms of exposure. The K-PERS-I and -II were developed based on the PPS using OR and ERS using RR, because their subcategories of risk factors were different. In addition, considering importance of childhood trauma in the development of psychosis, ${ }^{14}$ subcategory and its cut-off of childhood trauma were differentially designed in the K-PERS-I and -II to reflect the data from self-rating scale and structured interview respectively. A manual for using the K-PERS was also developed and is available on request.

\section{Pilot K-PERS data for patients with SSDs and HCs}

Pilot K-PERS data were obtained from patients with SSDs, including SZ, schizoaffective disorder, schizophreniform disorder, and psychotic disorder not otherwise specified ( $n=130$ and 217 for the K-PERS-I and -II, respectively), participating in the KEPS, ${ }^{19}$ and from HCs ( $n=126$ and 154, respectively). HCs were recruited via advertisements and interviewed using the Structured Clinical Interview for DSM, Non-Patient Edition (SCID-NP). ${ }^{20} \mathrm{~A}$ requirement for study inclusion was no previous or current psychiatric disorders, neurological disorders, or significant medical conditions. Controls having a firstdegree relative with a psychiatric disorder were also excluded. The sample sizes differed according to the K-PERS version because we only included patients and HCs with complete data for the version used. The total and subdomain scores on the K-PERS were compared between patients and HCs. The relationships of K-PERS scores with duration of illness and Positive and Negative Syndrome Scale (PANSS) scores ${ }^{21}$ were also examined. All participants provided written informed consent in accordance with a protocol approved by the Ethics Committee of Jeonbuk National University Hospital (approval number CUH 2014-11-002).

\section{RESULTS}

\section{K-PERS-I}

For the K-PERS, low parental SES was defined as receiving medical aid at the time of the respondent's birth. Urbanicity was considered present when a person was raised in a city for more than $50 \%$ of their early life (from birth to 12 years old). Adult life events referred to at least two adverse events including living alone, financial hardship, and difficulties in social relationships and occupational or academic functioning, experienced at the age of $\geq 19$ years at least 6 months prior to the development of psychotic symptoms. The PP values for paternal age, parental SES, urbanicity, childhood trauma, and clinical high risk for psychosis were acquired from official Korean data. However, as we did not find an appropriate data source for adult adverse life events, the same PP values used for the PPS $^{22}$ were applied. As the cutoffs and contents of the PPS are relatively simple, we named the developed tool the K-PERS-I (Table 1).

\section{K-PERS-II}

To accurately determine parental SES, a 7-point scale has been devised, on which low SES is reflected by on the presence of six factors (father's/mother's income in the lowest quintile, father/mother unemployed or outside the labor market, and father's/mother's highest educational level less than high school). ${ }^{23}$ As PP values were provided for each individual category but not for the summed score, six factors reflecting parental SES were included in the K-PERS-II. Because no Korean PP data for father's income in the lowest quintile and father unemployed or outside the labor market were available, data from Byrne et $\mathrm{al}^{24}$ were adopted. Urbanicity was roughly categorized as "metropolitan city," "city," or "county," as no Korean data on population density were available. In the ERS, childhood trauma was only categorized as exposed or nonexposed. However, considering its increasing importance in Korean society, more detailed categorizations ${ }^{25}$ were used for the K-PERS-II. Although the references cited ${ }^{18,22}$ in the PPS for recent life events are the same, using different RR values yielded different scores ( 3 and -2 for exposure and non-exposure) compared to those (5.5 and -2 for exposure and non-exposure) in the PPS. It is of note that parental SES and recent life events were not included in the original ERS. The tool developed based on the ERS was named the K-PERS-II (Table 2).

\section{Comparison of K-PERS-I -II scores between patients with SSDs and HCs}

For the K-PERS-I, we only calculated the scores for the first five factors listed in Table 1 because the sixth factor, clinical high risk for psychosis, was not applicable. The demographic 


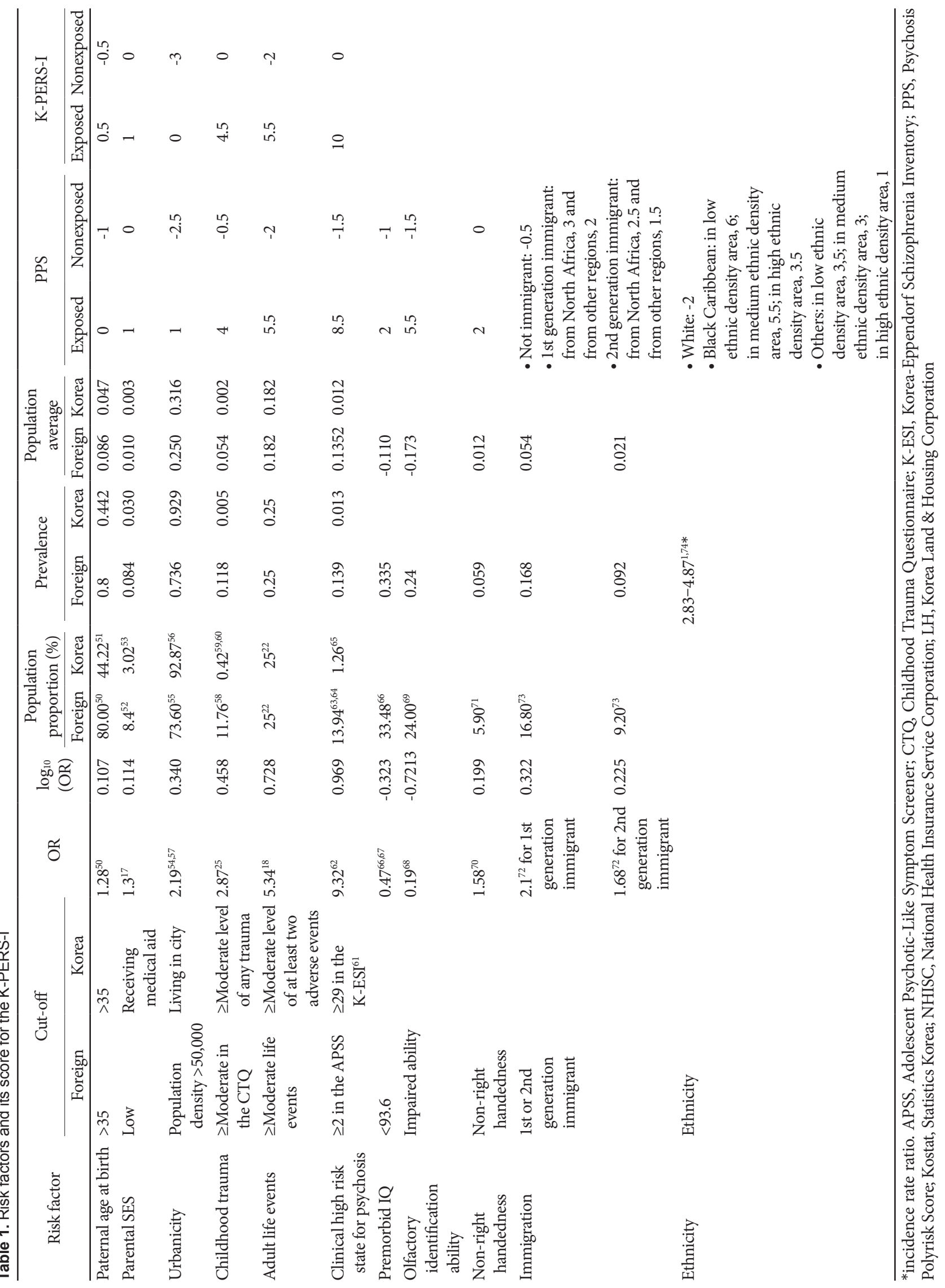




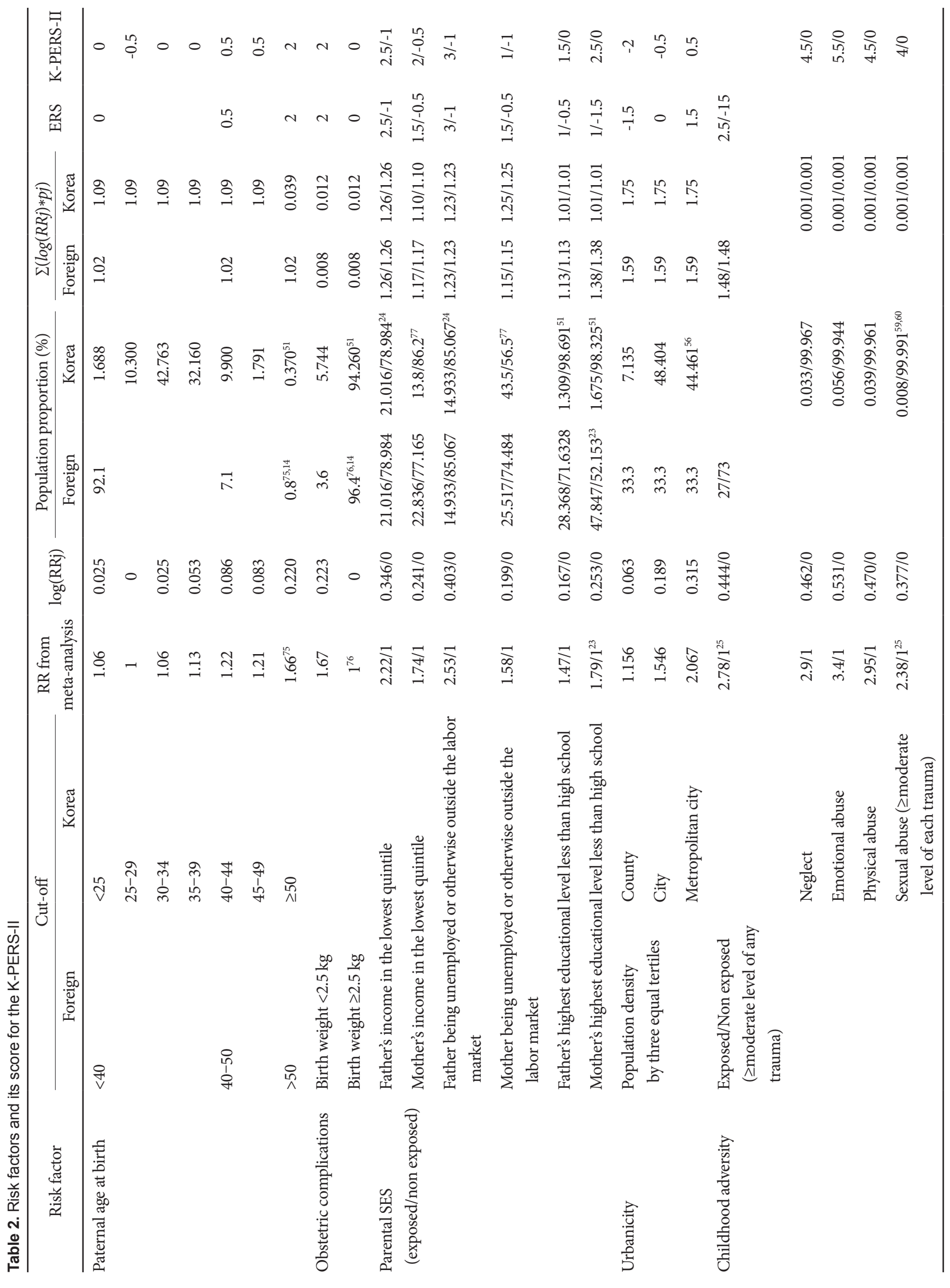




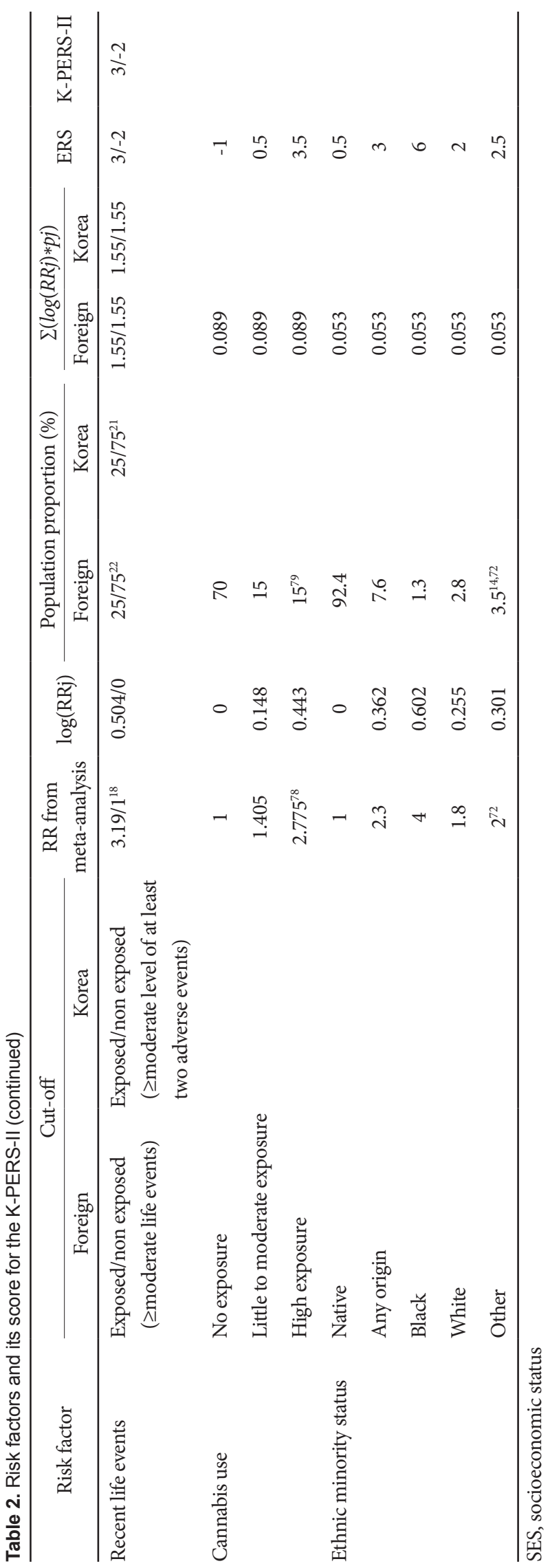

data (age, sex, and education) did not differ between patients and HCs according to the K-PERS-I and -II scores. The scores for all five factors of the K-PERS-I were significantly different between the two groups (Table 3). The total K-PERS-I and adult life events scores showed significant negative correlations with the negative symptom factor score of the PANSS (Table 4). For the K-PERS-II, only three factors were significantly different between the two groups (Table 5). The total K-PERS-II score exhibited a significant negative correlation with the negative symptom factor score of the PANSS (Table 6).

\section{DISCUSSION}

Among modern psychiatry disciplines, biological psychiatry has been the dominant research field, especially genetic and brain imaging studies. Most researchers in the field of molecular genetics now believe that many genes are involved (the polygenic theory) in the development of SZ, so have abandoned the single-gene approach. ${ }^{26,27}$ Even the PRS, a measure based on a set of genetic variants as risk factors, has limited value for predicting SZ symptoms and cognitive phenotypes $(<0.7 \%))^{28}$ Brain imaging studies of SZ have shown remarkable progress. Two major hypotheses developed based on these studies are that $\mathrm{SZ}$ is a neurodevelopmental disorder, ${ }^{29}$ and that it reflects disrupted brain connectivity (dysconnectivity hypothesis. ${ }^{30}$ However, with regard to the clinical utility of structural MRI (sMRI), one meta-analysis found no evidence to support diagnosing SZ (as opposed to other psychotic disorders) based on the pattern of brain changes revealed by voxel-based morphometry (VBM) studies of patients with first-episode psychosis. ${ }^{31}$ The specificity of altered functional connectivity (FC) to $\mathrm{SZ}$ is being questioned. ${ }^{32-34}$ Furthermore, it should be noted that exercise $^{35,36}$ and meditation ${ }^{37}$ can affect resting-state FC.

Numerous large-scale population-based studies have reported associations between various environmental factors and the prevalence of psychosis and psychotic symptoms. ${ }^{38}$ The environmental factors include childhood adversity, poverty, migration stress, social isolation, social defeat, mother in poor health, early loss of parents due to death or abandonment, witnessing interparental violence, dysfunctional parenting (such as affectionless overcontrol and communication deviance), poor nutrition and stress during pregnancy, racism, and war trauma. ${ }^{39}$ In contrast to the small amount of variance explained by genetic studies, the amount explained by environmental risk factors is quite high, at $33 \%$ of the estimated population attributable risk. ${ }^{25}$ As in genetic studies, specificity regarding the roles of various environmental factors in the development of SZ remains a critical issue that has yet to be resolved; taking account of multiple environmental risk factors is important, similar to the PRS for genetic factors. The development of comprehensive 
measures assessing environmental factors in SZ is crucial for enhancing psychosocial understanding of the disease and obtaining holistic viewpoints from patients. In addition, it should be noted that some environmental factors are amenable to psychosocial intervention and education.

In the case of the PRS, many studies have explored its predictive value with respect to conversion in persons at clinical high risk, ${ }^{40}$ its discriminative ability in case (first-episode psychosis)-control studies, ${ }^{41}$ and its ability to predict antipsychotic efficacy in first-episode psychosis. ${ }^{42}$ On the other hand, research using measures assessing polyenvironmental risk factors is limited. The PERS can predict conversion to psychosis in individuals with familial high risk of SZ. ${ }^{13}$ In individuals with SZ, Stepniak et al. ${ }^{43}$ reported an association between an increased number of environmental risk factors and age at SZ onset. Cougnard et al. ${ }^{44}$ reported an interaction effect between exposure to three risk factors (cannabis use, childhood trauma, and urbanicity) and baseline psychotic experiences on the likelihood of psychotic symptoms 3 years later in the general population. To date, no study has investigated the relationships between polyenvironmental risk factors and clinical outcomes in SZ. In the present study, we found that total scores on the K-PERS-I and -II differentiated between patients with SSDs and HCs, suggesting their predictive utility for both patients with ambiguous clinical features and the general population.

Table 3. Comparison of K-PERS-I between patients and healthy controls

\begin{tabular}{lccc}
\hline & $\begin{array}{c}\text { Patients } \\
(\mathrm{N}=217)\end{array}$ & $\begin{array}{c}\text { Healthy controls } \\
(\mathrm{N}=154)\end{array}$ & p-value \\
\hline Paternal age at birth & $-0.24(0.44)$ & $-0.37(0.34)$ & 0.0011 \\
Parental SES & $0.40(0.49)$ & $0.17(0.37)$ & $2.6 \mathrm{E}-07$ \\
Urbanicity & $-0.80(1.33)$ & $-0.27(0.86)$ & $4.9 \mathrm{E}-06$ \\
Childhood trauma & $2.90(2.16)$ & $0.88(1.79)$ & $<0.05$ \\
Adult life events & $1.77(3.76)$ & $-1.37(2.09)$ & $<0.05$ \\
Total & $4.04(4.99)$ & $-0.96(3.15)$ & $<0.05$ \\
\hline
\end{tabular}

Values are presented as mean (SD, standard deviation). K-PERS, Korea Polyenvironmental Risk Score; SES, socioeconomic status
Of note, some items on the K-PERS-I and -II did not predict participants' status (case vs. control), raising questions about their validity. As the cutoff criteria for factors in the K-PERS-II are more refined, it is recommended that it be used when detailed data are available. For example, when childhood adversity is measured with a self-rating scale, such as the Childhood Trauma Questionnaire (CTQ) ${ }^{45}$ or Early Trauma InventorySelf Report (ETI), ${ }^{46}$ the K-PERS-I is recommended; the KPERS-II is recommended when a structured interview is used. Correlation analyses identified significant negative associations of the K-PERS-I and -II scores with negative symptom factor scores. This suggests that multiple environmental risk factors may have an impact on the development of negative symptoms in SSDs. Given that negative symptoms are often viewed as having a neurobiological basis, ${ }^{47}$ this finding is meaningful, although the underlying mechanisms remain to be investigated.

Several limitations of this study should be mentioned. First, we decided to use OR and RR data from Western studies on relationships between polyenvironmental risk factors and SZ,

Table 5. Comparison of K-PERS-II between patients and healthy controls

\begin{tabular}{lccc}
\hline & $\begin{array}{c}\text { Patient group } \\
(\mathrm{N}=130)\end{array}$ & $\begin{array}{c}\text { Control group } \\
(\mathrm{N}=126)\end{array}$ & p-value \\
\hline Paternal age at birth & $0.04(0.13)$ & $0.02(0.10)$ & 0.30 \\
Obstetric complications & $0.12(0.48)$ & $0.08(0.39)$ & 0.43 \\
Parental SES & $2.82(3.47)$ & $2.94(3.59)$ & 0.80 \\
Urbanicity & $-0.61(0.93)$ & $-0.85(0.63)$ & 0.02 \\
Childhood adversity & $5.84(5.51)$ & $1.94(3.74)$ & $2.1 \mathrm{E}-10$ \\
$\quad$ Emotional abuse & $2.88(2.76)$ & $0.87(2.02)$ & $2.0 \mathrm{E}-10$ \\
$\quad$ Neglect & $1.14(1.97)$ & $0.64(1.58)$ & 0.0258 \\
$\quad$ Physical abuse & $1.45(2.11)$ & $0.36(1.22)$ & $7.5 \mathrm{E}-07$ \\
$\quad$ Sexual abuse & $0.37(1.16)$ & $0.06(0.50)$ & 0.01 \\
Recent life events & $0.96(2.47)$ & $-0.41(2.34)$ & $7.5 \mathrm{E}-06$ \\
Total & $9.18(7.16)$ & $3.71(6.74)$ & $1.4 \mathrm{E}-09$ \\
\hline
\end{tabular}

Values are presented as mean (SD, standard deviation). K-PERS, Korea Polyenvironmental Risk Score; SES, socioeconomic status

Table 4. Correlation of the K-PERS-I with DI and PANSS

\begin{tabular}{lccccc}
\hline & \multirow{2}{*}{ DI } & \multicolumn{4}{c}{ PANSS } \\
\cline { 3 - 6 } & & Total & Positive symptoms & Negative symptoms & General psychopathology \\
\hline Paternal age at birth & $-0.02(0.79)$ & $-0.06(0.36)$ & $-0.06(0.36)$ & $-0.05(0.48)$ & $-0.05(0.43)$ \\
Parental SES & $0.09(0.21)$ & $0.01(0.94)$ & $0.00(0.97)$ & $-0.02(0.82)$ & $0.02(0.76)$ \\
Urbanicity & $-0.09(0.19)$ & $-0.02(0.73)$ & $-0.04(0.53)$ & $0.00(0.96)$ & $-0.02(0.73)$ \\
Childhood trauma & $0.05(0.46)$ & $-0.03(0.67)$ & $0.08(0.22)$ & $-0.11(0.12)$ & $-0.04(0.58)$ \\
Adult life events & $0.08(0.22)$ & $-0.11(0.09)$ & $-0.05(0.5)$ & $-0.14(0.05)$ & $-0.11(0.12)$ \\
Total & $0.07(0.32)$ & $-0.11(0.11)$ & $-0.02(0.82)$ & $-0.15(0.03)$ & $-0.11(0.12)$ \\
\hline
\end{tabular}

Values are presented as correlation coefficient (p-value). K-PERS, Korea Polyenvironmental Risk Score; DI, duration of illness; PANSS, Positive and Negative Syndrome Scale; SES, socioeconomic status 
Table 6. Correlation of the K-PERS-II with DI and PANSS

\begin{tabular}{lccccc}
\hline & & \multicolumn{3}{c}{ PANSS } \\
\cline { 3 - 6 } & DI & Total & $\begin{array}{c}\text { Positive } \\
\text { symptoms }\end{array}$ & $\begin{array}{c}\text { Negative } \\
\text { symptoms }\end{array}$ & $\begin{array}{c}\text { General } \\
\text { psychopathology }\end{array}$ \\
\hline Father's age at patient's birth & & $-0.05(0.61)$ & $-0.02(0.9)$ & $-0.1(0.26)$ & $-0.01(0.88)$ \\
Obstetrics and perinatal complication & $0.14(0.11)$ & $-0.00(0.99)$ & $0.04(0.67)$ & $-0.06(0.53)$ & $0.01(0.88)$ \\
SES & $0.08(0.39)$ & $-0.13(0.13)$ & $-0.08(0.37)$ & $-0.16(0.08)$ & $-0.11(0.21)$ \\
Urbanization & $-0.1(0.28)$ & $-0.04(0.62)$ & $-0.12(0.18)$ & $0.05(0.58)$ & $-0.05(0.58)$ \\
Childhood trauma & $0.0(0.96)$ & $-0.04(0.67)$ & $0.01(0.88)$ & $-0.12(0.17)$ & $-0.00(0.98)$ \\
$\quad$ Emotional abuse & $0.02(0.76)$ & $-0.01(0.95)$ & $0.08(0.35)$ & $-0.13(0.13)$ & $0.03(0.75)$ \\
$\quad$ Neglect & $0.06(0.53)$ & $-0.06(0.53)$ & $-0.07(0.44)$ & $-0.07(0.43)$ & $-0.02(0.81)$ \\
$\quad$ Physical abuse & $-0.09(0.3)$ & $-0.04(0.67)$ & $0.01(0.95)$ & $-0.09(0.33)$ & $-0.02(0.83)$ \\
$\quad$ Sexual abuse & $0.03(0.75)$ & $-0.01(0.94)$ & $-0.03(0.76)$ & $0.02(0.85)$ & $-0.01(0.92)$ \\
Difficulties of adulthood & $-0.04(0.64)$ & $-0.1(0.27)$ & $-0.14(0.13)$ & $-0.05(0.56)$ & $-0.07(0.39)$ \\
Total score & $0.02(0.80)$ & $-0.13(0.13)$ & $-0.09(0.32)$ & $-0.18(0.03)$ & $-0.09(0.32)$ \\
\hline
\end{tabular}

Values are presented as correlation coefficient (p-value). K-PERS, Korea Polyenvironmental Risk Score; DI, duration of illness; PANSS, Positive and Negative Syndrome Scale; SES, socioeconomic status

so the K-PERS may not truly reflect Koreans' experiences. To address this shortcoming, large-scale epidemiologic studies investigating relationships between environmental risk factors and psychosis should be performed. Second, ethnicity and immigration were not incorporated into the K-PERS. Due to increasing immigration from other Asian countries, this should be considered in future versions of the K-PERS. Third, conflict and stress related to family members was not considered in the K-PERS; however, this factor was also not included in the ERS, PERS, and PPS due to a lack of evidence. Nevertheless, several studies have suggested an important role of the family environment in the development of psychosis, ${ }^{48}$ and in poor prognosis. ${ }^{49}$ When sufficient evidence accumulates, this factor will likely be considered as one of the main polyenvironmental risk factors for SZ. Lastly, to confirm validity of the K-PERS, future study with large sample size is warranted. Despite these caveats, the K-PERS is the first comprehensive tool developed based on PP data from Korean studies, and successfully measures polyenvironmental risk factors for psychosis. Using pilot data, the K-PERS was able to differentiate between patients with SSDs and HCs. Further research is warranted to examine the relationship of K-PERS scores with clinical outcomes in psychosis and SZ.

\section{Supplementary Materials}

The online-only Data Supplement is available with this article at https://doi.org/10.30773/pi.2021.0328.

\section{Availability of Data and Material}

The datasets generated or analyzed during the study are available from the corresponding author on reasonable request.

\section{Conflicts of Interest}

Sung-Wan Kim and Euitae Kim, a contributing editor of the Psychiatry Investigation, was not involved in the editorial evaluation or decision to publish this article. All remaining authors have declared no conflicts of interest.

\section{Author Contributions}

Conceptualization: Young-Chul Chung. Data curation: Yan-Hong Piao, Bong-Ju Lee, Sung-Wan Kim, Jung-Jin Kim. Formal analysis: Je-Chun Yu, Kyu-Young Lee, Seung-Hwan Lee. Funding acquisition: Young-Chul Chung, Seung-Hee Won. Investigation: Seung-Hyun Kim, Eui-Tae Kim. Methodology: Bong-Ju Lee, Clara Tammy Kim. Project administration: Young-Chul Chung. Resources: Shi-Hyun Kang. Software: Fatima Zahra Rami. Supervision: Young-Chul Chung. Validation: Paolo Fusar-Poli. Visualization: Dominic Oliver. Writing_-original draft: Young-Chul Chung, Eun Jin Jeon. Writing_review \& editing: Young-Chul Chung, Eun Jin Jeon.

\section{ORCID iDs}

Eun-Jin Jeon Shi-Hyun Kang Yan-Hong Piao Sung-Wan Kim Jung-Jin Kim Bong-Ju Lee Je-Chun Yu Kyu-Young Lee Seung-Hee Won Seung-Hwan Lee Seung-Hyun Kim Eui-Tae Kim Clara Tammy Kim Dominic Oliver Paolo Fusar-Poli Fatima Zahra Rami Young-Chul Chung

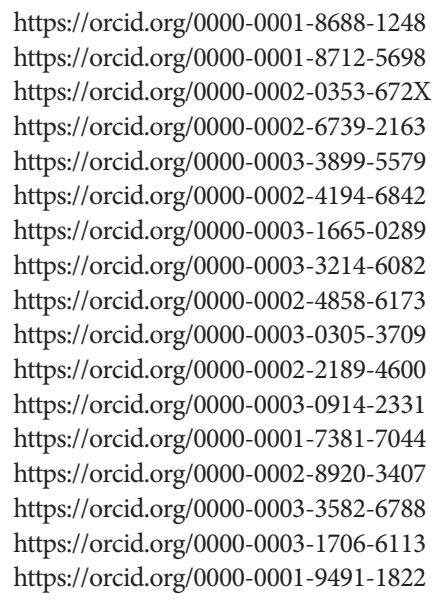

\section{Funding Statement}

This study was supported by grants from the Korean Mental Health Technology R\&D Project, Ministry of Health and Welfare, Republic of Korea (HL19C0015), and the Korea Health Technology R\&D Project through 
the Korea Health Industry Development Institute (KHIDI), funded by the Ministry of Health and Welfare, Republic of Korea (HI18C2383).

\section{REFERENCES}

1. Kirkbride JB, Errazuriz A, Croudace TJ, Morgan C, Jackson D, Boydell $\mathrm{J}$, et al. Incidence of schizophrenia and other psychoses in England, 1950-2009: a systematic review and meta-analyses. PLoS One 2012;7: e31660.

2. Thara R. Twenty-year course of schizophrenia: the Madras Longitudinal Study. Can J Psychiatry 2004;49:564-569.

3. Wiersma D, Nienhuis FJ, Slooff CJ, Giel R. Natural course of schizophrenic disorders: a 15-year followup of a Dutch incidence cohort. Schizophr Bull 1998;24;75-85.

4. Laursen TM, Munk-Olsen T, Vestergaard M. Life expectancy and cardiovascular mortality in persons with schizophrenia. Curr Opin Psychiatry 2012;25:83-88.

5. Kendler KS. Overview: a current perspective on twin studies of schizophrenia. Am J Psychiatry 1983;140:1413-1425.

6. Lichtenstein P, Yip BH, Bjork C, Pawitan Y, Canno TD, Sullivan PF, et al. Common genetic determinants of schizophrenia and bipolar disorder in Swedish families: a population-based study. Lancet 2009;373: 234-239.

7. Sullivan PF, Kendler KS, Neale MC. Schizophrenia as a complex trait: evidence from a meta-analysis of twin studies. Arch Gen Psychiatry 2003;60:1187-1192.

8. Schizophrenia Working Group of the Psychiatric Genomics Consortium. Biological insights from 108 schizophrenia-associated genetic loci. Nature 2014;511:421-427.

9. International Schizophrenia Consortium; Purcell SM, Wray NR, Stone JL, Visscher PM, O’Donovan MC, et al. Common polygenic variation contributes to risk of schizophrenia and bipolar disorder. Nature 2009; 460:748-752.

10. Maher B. Personal genomes: the case of the missing heritability. Nature 2008;456:18-21.

11. Brown AS. The environment and susceptibility to schizophrenia. Prog Neurobiol 2011;93:23-58.

12. Davis C, Segre G, Estrade A, Radua J, Micheli A, Provenzani U, et al. Prenatal and perinatal risk and protective factors for psychosis: a systematic review and meta-analysis. Lancet Psychiatry 2020;7:399-410.

13. Padmanabhan JL, Shah JL, Tandon N, Keshavan MS. The "polyenviromic risk score": aggregating environmental risk factors predicts conversion to psychosis in familial high-risk subjects. Schizophr Res 2017; 181:17-22.

14. Vassos E, Sham P, Kempton M, Trotta A, Stilo SA, Gayer-Anderson C, et al. The Maudsley environmental risk score for psychosis. Psychol Med 2020;50:2213-2220.

15. Oliver D, Radua J, Reichenberg A, Uher R, Fusar-Poli P. Psychosis Polyrisk Score (PPS) for the detection of individuals at-risk and the prediction of their outcomes. Front Psychiatry 2019;10:174.

16. Oliver D, Spada G, Englund A, Chesney E, Radua J, Reichenberg A, et al. Real-world digital implementation of the Pychosis Polyrisk Score (PPS): a pilot feasibility study. Schizophr Res 2020;226:176-183.

17. Kwok W. Is there evidence that social class at birth increases risk of psychosis? A systematic review. Int J Soc Psychiatry 2014;60:801-808.

18. Beards S, Gayer-Anderson C, Borges S, Dewey M, Fisher H, Morgan C. Life events and psychosis: a review and meta-analysis. Schizophr Bull 2013;39:740-747.

19. Kim SW, Lee BJ, Kim JJ, Yu JC, Lee KY, Won SH, et al. Design and methodology of the Korean early psychosis cohort study. Psychiatry Investig 2017;14:93-99.

20. Hahn OS, Ahn JH, Song SH, Cho MJ, Kim JK, Bae JN, et al. Development of Korean version of structured clinical interview schedule for DSM-IV Axis I disorder: interrater reliability. J Korean Neuropsychiatr Assoc 2000;39:362-372.
21. Kay SR, Fiszbein A, Opler LA. The positive and negative syndrome scale (PANSS) for schizophrenia. Schizophr Bull 1987;13:261-276.

22. Johns L, Cannon M, Singleton N, Murray RM, Farrell M, Brugha T, et al. Prevalence and correlates of self-reported psychotic symptoms in the British population. Br J Psychiatry 2004;185:298-305.

23. Agerbo E, Sullivan PF, Vilhjálmsson BJ, Pedersen CB, Mors O, Børglum $\mathrm{AD}$, et al. Polygenic risk score, parental socioeconomic status, family history of psychiatric disorders, and the risk for schizophrenia: a Danish population-based study and meta-analysis. JAMA Psychiatry 2015; 72:635-641.

24. Byrne M, Agerbo E, Eaton WW, Mortensen PB. Parental socio-economic status and risk of first admission with schizophrenia: a Danish national register based study. Soc Psychiatry Psychiatr Epidemiol 2004; 39:87-96.

25. Varese F, Smeets F, Drukker M, Lieverse R, Lataster T, Viechtbauer W, et al. Childhood adversities increase the risk of psychosis: a meta-analysis of patient-control, prospective- and cross-sectional cohort studies. Schizophr Bull 2012;38:661-671.

26. Moldin SO, Gottesman II. At issue: genes, experience, and chance in schizophrenia - positioning for the 21st century. Schizophr Bull 1997; 23:547-61.

27. Portin P, Alanen Y. A critical review of genetic studies of schizophrenia. Acta Psychiatr Scand 1997;95:73-80.

28. Mistry S, Harrison JR, Smith DJ, Escott-Price V, Zammit S. The use of polygenic risk scores to identify phenotypes associated with genetic risk of schizophrenia: Systematic review. Schizophr Res 2018;197:2-8.

29. Owen MJ, O’Donovan MC, Thapar A, Craddock N. Neurodevelopmental hypothesis of schizophrenia. Br J Psychiatry 2011;198:173-175.

30. Friston Karl, Brown HR, Siemerkus J, Stephan KE. The dysconnection hypothesis (2016) Schizophr Res 2016;176:83-94.

31. Palaniyappan L, Maayan N, Bergman H, Davenport C, Adams CE, Soares-Weiser K. Voxel-based morphometry for separation of schizophrenia from other types of psychosis in first episode psychosis. Cochrane Database Syst Rev 2015;2015:CD011021.

32. Argyelan M, Ikuta T, DeRosse P, Braga RJ, Burdick KE, John M, et al. Resting-state fMRI connectivity impairment in schizophrenia and bipolar disorder. Schizophr Bull 2014;40:100-110.

33. Huang CC, Luo Q, Palaniyappan L, Yang AC, Hung CC, Chou KH, et al. Transdiagnostic and illness-specific functional dysconnectivity across schizophrenia, bipolar disorder, and major depressive disorder. Biol Psychiatry Cogn Neurosci Neuroimaging 2020;5:542-553.

34. Li C, Xu K, Dong M, Wei Y, Duan J, Han S, et al. Shared dynamic functional connectivity across schizophrenia, bipolar disorder and major depressive disorder. BioRxiv; 2019.

35. Greeley B, Chau B, Jones CB, Neva JL, Kraeutner SN, Campbell KL, et al. Multiple bouts of high-intensity interval exercise reverse age-related functional connectivity disruptions without affecting motor learning in older adults. BioRxiv; 2021.

36. Rajab AS, Crane DE, Middleton LE, Robertson AD, Hampson M, Maclntosh BJ. A single session of exercise increases connectivity in sensorimotor-related brain networks: a resting-state fMRI study in young healthy adults. Front Hum Neurosci 2014;8:625.

37. Zhang Z, Luh WM, Duan W, Zhou GD, Weinschenk G, Anderson $\mathrm{AK}$, et al. Longitudinal effects of meditation on brain restingstate functional connectivity. Sci Rep 2021;11:11361.

38. Read J, Fosse R, Moskowitz AP. The traumagenic neurodevelopmental model of psychosis revisited. Neuropsychiatry 2014;4:65-79.

39. Read J, Bentall RP, Fosse R. Time to abandon the bio-bio-bio model of psychosis: exploring the epigenetic and psychological mechanisms by which adverse life events lead to psychotic symptoms. Epidemol Psichiatr Soc 2009;18:299-310.

40. Perkins D, Loohuis LO, Barbee J, Ford J, Jeffries C, Addington J, et al. Polygenic risk score contribution to psychosis prediction in a target population of persons at clinical high risk. Am J Psychiatry 2020;177: 155-163. 
41. Vassos E, Di Forti M, Coleman J, Iyegbe C, Prata D, Euesden J, et al. An examination of polygenic score risk prediction in individuals with first episode psychosis. Biol Psychiatry 2017;81:470-477.

42. Zhang JP, Robinson D, Yu J, Gallego J, Fleischhacker WW, Kahn RS, et al. Schizophrenia polygenic risk score as a predictor of antipsychotic efficacy in first-episode psychosis. Am J Psychiatry 2019;176:21-28.

43. Stepniak B, Papiol S, Hammer C, Ramin A, Everts S, Hennig L, et al. Accumulated environmental risk determining age at schizophrenia onset: a deep phenotyping-based study. Lancet Psychiatry 2014;1:444-453.

44. Cougnard A, Marcelis M, Myin-Germeys I, De Graaf R, Vollebergh W, Krabbendam L, et al. Does normal developmental expression of psychosis combine with environmental risk to cause persistence of psychosis? A psychosis proneness-persistence model. Psychol Med 2007; 37:513-527.

45. Bernstein DP, Stein JA, Newcomb MD, Walker E, Pogge D, Ahluvalia $\mathrm{T}$, et al. Development and validation of a brief screening version of the childhood trauma questionnaire. Child Abuse Negl 2003;27:169-190.

46. Bremner JD, Bolus R, Mayer EA. Psychometric properties of the early trauma inventory-self report. J Nerv Ment Dis 2007;195:211-218.

47. Correll CU, Schooler NR. Negative symptoms in schizophrenia: a review and clinical guide for recognition, assessment, and treatment. Neuropsychiatr Dis Treat 2020;16:519-534.

48. González-Pinto A, Ruiz de Azúa S, Ibáñez B, Otero-Cuesta S, CastroFornieles J, Graell-Berna, et al. Can positive family factors be protective against the development of psychosis? Psychiatry Res 2011;186:28-33.

49. Myin-Germeys I, van Os J, Schwartz JE, Stone AA, Delespaul PA. Emotional reactivity to daily life stress in psychosis. Arch Gen Psychiatry 2001;58:1137-1144.

50. Torrey EF, Buka S, Cannon TD, Goldstein JM, Seidman LJ, Liu T, et al. Paternal age as a risk factor for schizophrenia: how important is it? Schizophr Res 2009;114:1-5.

51. Statistics Korea. Population Trend Survey. Daejeon: Statistics Korea; 2015.

52. Wicks S, Hjern A, Gunnell D, Lewis G, Dalman C. Social adversity in childhood and the risk of developing psychosis: a National Cohort Study. Am J Psychiatry 2005;162:1652-1657.

53. National Health Insurance Service Corporation, Health Insurance Review \& Assessment Service. Medical Aid Statistics. Wonju-si: Health Insurance Review \& Assessment Service National Health Insurance Service; 2015

54. Laurens KR, Luo L, Matheson SL, Carr VJ, Raudino A, Harris F, et al. Common or distinct pathways to psychosis? A systematic review of evidence from prospective studies for developmental risk factors and antecedents of the schizophrenia spectrum disorders and affective psychoses. BMC Psychiatry 2015;15:205.

55. United Nations, Department of Economic and Social Affairs, Population Division (2015). World Urbanization Prospects: The 2014 Revision. New York: United Nations; 2015.

56. Korea Land \& Housing Corporation. Statistics for Urban Planning. Sejong: Ministry of Land, Infrastructure and Transport, Korea Land \& Housing Corporation; 2018.

57. Matheson SL, Shepherd AM, Laurens KR, Carr VJ. A systematic metareview grading the evidence for non-genetic risk factors and putative antecedents of schizophrenia. Schizophr Res 2011;133:133-142.

58. Nierop M, Os J, Gunther N, Myin-Germeys I, Graaf R, Have M, et al. Phenotypically continuous with clinical psychosis, discontinuous in need for care: evidence for an extended psychosis phenotype. Schizophr Bull 2012;38:231-238.

59. Statistics Korea. Future Population Estimation. Daejeon: Statistics Korea; 2017.

60. Ministry of Health and Welfare. Child Abuse \& Neglect Korea. Seoul: Ministry of Health and Welfare, National Child Protection Agency; 2017.
61. Chung YC, Kang NI, Im YJ, Kim SW, Cho IH, Lee YM, et al. Validation of the Korean version of the Eppendorf Schizophrenia Inventory as a screening measure to detect adolescents at ultra-high-risk for psychosis. Early Interv Psychiatry 2013;7:71-79.

62. Fusar-Poli P, Cappucciati M, Rutigliano G, Schultze-Lutter F, Bonoldi I, Borgwardt S, et al. At risk or not at risk? A meta-analysis of the prognostic accuracy of psychometric interviews for psychosis prediction. World Psychiatry 2015;14:322-332.

63. Ising HK, Veling W, Loewy RL, Rietveld MW, Rietdijk K, Dragt S, et al. The validity of the 16-item version of the Prodromal Questionnaire (PQ-16) to screen for ultra high risk of developing psychosis in the general help-seeking population. Schizophr Bull 2012; 38:1288-1296.

64. Schimmelmann BG, Michel C, Martz-Irngartinger A, Linder C, Schultze-Lutter F. Age matters in the prevalence and clinical significance of ultra-high-risk for psychosis symptoms and criteria in the general population: findings from the BEAR and BEARS-kid studies. World Psychiatry 2015;14:189-197.

65. Kang NI, Park TW, Yang JC, Oh KY, Shim SH, Chung YC. Prevalence and clinical features of Thought-Perception-Sensitivity Symptoms: results from a community survey of Korean high school students. Psychiatry Res 2012;198:501-508.

66. Khandaker GM, Barnett JH, White IR, Jones PB. A quantitative metaanalysis of population-based studies of premorbid intelligence and schizophrenia Schizophr Res 2011;132:220-227.

67. Woodberry KA, Giuliano AJ, Seidman LJ. Premorbid IQ in schizophrenia: a meta-analytic review. Am J Psychiatry 2008;165:579-587.

68. Moberg P, Kamath V, Marchetto D, Calkins ME, Doty RL, Hahn CG, et al. Meta-analysis of olfactory function in schizophrenia, first-degree family members, and youths at-risk for psychosis. Schizophr Bull 2014; 40:50-59.

69. Doty RL. The Smell Identification Test Administration Manual. New Jersey: Sensonics International; 2013.

70. Hirnstein M, Hugdahl K. Excess of non-right-handedness in schizophrenia: meta-analysis of gender effects and potential biases in handedness assessment. Br J Psychiatry 2014;205:260-267.

71. Vuoksimaa E, Koskenvuo M, Rose RJ, Kaprio J. Origins of handedness: a nationwide study of 30,161 adults. Neuropsychologia 2009;47:12941301.

72. Bourque F, van der Ven E, Malla A. A meta-analysis of the risk for psychotic disorders among first- and second-generation immigrants. Psychol Med 2011;41:897-910.

73. Eurostat: European Union Labour Force Survey. Luxembourg: Publications Office of the European Union; 2014.

74. Tortelli A, Errazuriz A, Croudace T, Morgan C, Murray RM, Jones PB, et al. Schizophrenia and other psychotic disorders in Caribbean-born migrants and their descendants in England: systematic review and meta-analysis of incidence rates, 1950-2013. Soc Psychiatry Psychiatr Epidemiol 2015;50:1039-1055.

75. Miller B, Messias E, Miettunen J, Alaräisänen A, Järvelin MR, Koponen $\mathrm{H}$, et al. Meta-analysis of paternal age and schizophrenia risk in male versus female offspring. Schizophr Bull 2011;37:1039-1047.

76. Cannon M, Jones PB, Murray RM. Obstetric complications and schizophrenia: historical and meta-analytic review. Am J Psychiatry 2002; 159:1080-1092.

77. Korea Institute for Health and Social Affairs. The National Survey on Fertility, Family Health and Welfare. Sejong: Korea Institute for Health and Social Affairs; 2018.

78. Marconi A, Di Forti M, Lewis CM, Murray RM, Vassos E. Meta-analysis of the association between the level of cannabis use and risk of psychosis. Schizophr Bull 2016;42:1262-1269.

79. European Drug Report: Trends and Developments. Luxembourg: EMCDDA; 2017. 